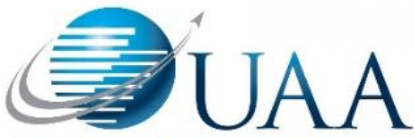

Collegiate Aviation Review

International

UNIVERSITY AVIATION

ASSOCIATION

\title{
Utilizing Organization Change Management Tools to Implement Safety Management System (SMS) Principles
}

\author{
Stephanie Brown \\ Purdue University \\ Brian Dillman \\ Purdue University
}

This paper presents an exploration of organizational change management tools and their usability to the Safety Management System (SMS) principles. The objective is to provide three tools to embrace the three dimensions of organizational change management, aiding leaders to adopt to a magnitude of different changes or initiatives while following the same blueprint consistently that promotes a positive safety culture and identifies new hazards or ineffective risk controls within that operation. With SMS becoming the aviation industry standard for safety program management it is vital to not only comply with the regulations set forth but to be able to embed functionality and adaptability within an operation in a systematic, explicit, and comprehensive way that can be applied consistently time and time again when a new hazard or ineffective risk control is identified within an organization. Addressing the actions required to comply with the regulations and leveraging the organizational change management dimensions will create enhanced sustainability and resilience for the overall safety program.

This exploration and design solution will create future research and analysis on the implementation of the guides in operation.

\section{Recommended Citation:}

Brown, S., \& Dillman, B. (2021). Utilizing organization change management tools to implement Safety

Management System (SMS) principles. Collegiate Aviation Review International, 39(2), 274-284.

Retrieved from http://ojs.library.okstate.edu/osu/index.php/CARI/article/view/8401/7690 


\section{Introduction}

\section{Problem Statement}

Safety Management System (SMS) contains four components (Safety Policy, Safety Risk Management, Safety Assurance, and Safety Promotion). When implemented into an operation, they create a robust safety culture and safety program. How an organization implements these components are left undefined by the regulators and open to creative interpretation by the operators and organizations. While this may first appear to be an ideal situation, this often fosters confusion, inconsistencies, and margins of error in the applications of the operator's programs which can lead to improper or nonfunctional mitigation controls into an operation. This can result in safety concerns such as injury or damage to people or equipment.

\section{Background}

Safety Management System (SMS) principles and organizational change management at their core focus on identifying changes, creating systematic approaches to change, and creating effective solutions for the operation with a people focus. Evidence of the linkage between SMS principles and organizational change management is described in their definitions. AC $120-$ 92B explains the purpose of Safety Management System (SMS) as:

An SMS is an organization-wide comprehensive and preventive approach to managing safety. An SMS includes a safety policy, formal methods for identifying hazards and mitigating risk, and promotion of a positive safety culture. An SMS also provides assurance of the overall safety performance of your organization. An SMS is intended to be designed and developed by your own people and should be integrated into your existing operations and business decision-making processes. The SMS will assist your organization's leadership, management teams, and employees in making effective and informed safety decisions. (FAA, 2015)

An article titled Innovation-Based Change Management (GAM, 2015) defines change management as "a systematic approach to dealing with change, both from the perspective of an organization and on the individual level...proactively addressing adapting to change, controlling change, and effecting change."

Applying organizational change management tools to implement SMS principles into an operation will create a solid framework for operators to successfully comply and articulate the needs of the program to the population while verifying critical aspects are completed consistently throughout the organization. 


\section{Literature Review}

\section{Safety Management System}

Understanding the makeup of SMS and its components is important to understand because it helps connect the framework of the guides to its effectiveness of complying with the SMS principles while embracing organizational change management dimensions that will be impactful for lasting change and acceptance in an organization.

Safety Management System (SMS) is becoming the industry standard throughout aviation. The program is now recognized by Joint Planning and Development Office (JPDO), International Civil Aviation Organization (ICAO), and Civil Aviation Authorities (CAA) (Safety Management System, 2016). The program focuses on integrating safety risk management and safety assurance concepts into repeatable and proactive systems, which results in risk identification, evaluation and mitigation, which plays a role in accident prevention. Operators benefit from using the program concepts (four components) and principles by having:

- A structured means of safety risk management decision making

- A means of demonstrating safety management capability before system failures occur

- Increased confidence in risk controls through structured safety assurance processes

- An effective interface for knowledge sharing between regulator and certificate holder

- A safety promotion framework to support a sound safety culture (Safety Management System, 2016)

SMS is structured by four components; Safety Policy, Safety Risk Management (SRM), Safety Assurance (SA), and Safety Promotion. By achieving the primary objectives of SMS and embracing the components, operators will have, "a well-structured SMS that provides a systematic, explicit, and comprehensive process for managing risks" (Ludwig, 2017).

\section{Safety Policy}

The Safety Policy component articulates the expectations of the policies and procedures and their related performance thresholds/goals, which creates the SMS program foundation. Even though senior leadership owns the commitment and responsibility to continually improve safety, it is up to all levels of the operation to be good stewards of the program and provide appropriate resources within their management levels authority. The output of this component is defined as,

"methods, processes and organizational structure needed to meet safety goals

- Establishes management commitment to safety performance through SMS

- Establishes clear safety objectives and commitment to manage those objectives

- Defines methods, process and organizational structure needed to meet safety goals

- Establishes transparency in the management of safety

○ Fully documented policy and processes

- Employee reporting and resolution system

- Accountability of management and employees 
- Builds upon the processes and procedures that already exist

- Facilitates cross-organization communication and cooperation" (Safety Management System, 2016).

\section{Safety Risk Management}

Understanding the makeup of SMS and its components is important to understand because it helps connect the framework of the guides to its effectiveness of complying with the SMS principles while embracing organizational change management dimensions that will be impactful for lasting change and acceptance in an organization.

Safety Risk Management (SRM) is comprised of five parts: system description, hazard identification, risk analysis, risk assessment, and risk control. This process is used to aid in the operator's practices for their operation, which identifies hazards and mitigating risks that are best suited for them. By participating in this activity, it ensures compliance with the commitment to "consider risk in their operations and to reduce it to an acceptable level" (Advisory Circular, 2015).

\section{Safety Assurance}

Safety Assurance is the evaluation component of an SMS program. Within the program, it assesses the effectiveness of implemented risk controls and supports the identification of new hazards being introduced. Aspects included in the component are as follows;

- SMS process management functions that systematically provide confidence that organization outputs meet or exceed safety requirements

- Ensures compliance with SMS requirements and FAA orders, standards, policies, and directives

$\circ$ Information Acquisitions

- Audits and evaluations

- Employee reporting

- Data Analysis

○ System Assessment

- Provides insight and analysis regarding methods/opportunities for improving safety and minimizing risk

- Existing assurance functions will continue to evaluate and improve service (Safety Management System, 2016).

\section{Safety Promotion}

The Safety Promotion component embeds safety culture aspects within which helps support a positive culture within all levels of an operation. Aspects of Safety Promotion include:

- Providing SMS training

- Advocating/strengthening a positive safety culture

- System and safety communication and awareness 
- Matching competency requirements to system requirements

- Disseminating safety lessons learned (Safety Management System, 2016).

Regardless of an employee's position within an organization, everyone has a vital role and a need to participate in promoting safety. It can be as simple as a one-on-one coaching conversation between a supervisor and an employee related to a potential safety hazard or as large as developing the training content that is deployed organization-wide educating on SMS training.

\section{Organizational Change Management}

Organizational change management can be broken up into three dimensions. Leaders of the intended change must balance the three dimensions in order to facilitate any type or magnitude of change to have a successful implementation or transition within an organization. The three dimensions are;

- Outcomes; developing and delivering clear outcomes

- Interests: mobilizing influence, authority, and power

- Emotions: enabling people and culture to adapt (Green, 2020).

When embedding the three dimensions into a structured process "Organizational change can be planned and managed through an understanding a set of sequential steps" (Burnes, 2009). This is one of the leading benefits of organizations adapting to these principles because they can be applied to a magnitude of different changes or initiatives while following the same blueprint consistently without investing design and assessment efforts for the overall framework of the change model.

\section{Change Management Tools}

Applying change management tools to SMS requirements will create a framework for organizations to successfully comply with regulatory requirements and articulate the needs of the program to employees while verifying critical aspects of work to be completed. A few tools to aid in this process are;

1. Project plan: identifies the milestones that must be completed to have a successful project outcome. This is especially beneficial when the change affects multiple unit groups, with work occurring simultaneously. This tool facilitates Safety Risk Management (SRM) by creating document the aids in identifying the system description listed out in the SMS requirements within the regulation and controlling risks within it.

2. Metrics and data collection: utilizing data throughout the project keeps the organization making decisions based on facts and not assumptions. This tool complies with the Safety Assurance component in SMS, where the requirement is to trend and review data of the operation on a regular basis to identify when a policy and procedure is becoming an ineffective risk control and needs to be processed through the Safety Risk Management process. 
3. Process mapping: shows a series of events that produce an end result that includes the who and the what and the workflow to achieve it. This tool applies to the Safety Risk Management (SRM) requirements and creates a system description and analysis and Safety Assurance.

\section{Problem Solving Approach}

The three tools (Safety Risk Management Documentation Tool, Safety Assurance Processing Mapping, and Communication Checklist) are created with utilizing the organizational change management dimensions' concepts with design requirements to meet 14 CFR Part 5 SMS regulatory requirements. The output creates workflows that the leaders can adopt to a magnitude of different changes or initiatives while following the same blueprint consistently that promotes a positive safety culture and identifies new hazards or ineffective risk controls within that operation.

\section{Safety Risk Management (SRM) Documentation Tool}

The SRM Documentation Tool was created to be utilized as a working document when participating in a SRM triggered from a new hazard or ineffective risk control in the operation that is a requirement of the Safety Risk Management component of the SMS. This tool is a resource developed under the Interests and Outcome of the organizational change management dimensions. By completing all aspects of the tool, it will verify the regulatory requirements are met, but it also acts as a user-friendly tool to assist the facilitator thru the overall process consistently regardless of the focus area, with mobilizing the stakeholders to develop business outcomes usually resulting in the form of new policy and procedures within the operation. Refer to Appendix A for the SRM Documentation Tool in its entirety.

To best leverage the tool, the facilitator of the SRM would complete the Overview section providing as much detail about the new hazard or ineffective risk control, its history within the operations and the trigger of the need to launch the SRM. Then send the document to all related stakeholders within the operation to provide insight from their Subject Matter Expert (SME) perspective. The request would include hazard conditions, data, and worst credible outcomes. Once this is complete, the facilitator would merge the information into a centralized document and facilitate a meeting where all the active participants would work through the rest of the SRM such as;

- Finalize hazard conditions

- Initial risk assessments

- Mitigation plans

- Document lessons learned

- Mitigated risk assessments

\section{Safety Assurance}

Process mapping is a powerful tool for the Safety Assurance component in SMS. This tool is a resource developed in Emotions and Outcomes of organizational change management 
dimensions. It showcases the required steps of the process and provides a way for different management level reviews to be embedded into the process in an easy-to-read and visual way for all facilitators and/or stakeholders to use when reviewing their safety performance data in a systemic way. Most commonly the data used in this process is de-identified safety reporting reports. This tool can also be used for promotional purposes to share how the data (i.e., safety reporting or metrics) is reviewed on a regular basis. Figure 1 depicts the steps included in the Safety Assurance process map.

A way to use this process is having the Safety Manager facilitate a monthly review (first Wednesday of the month) and analysis of safety metrics and related operational metrics with the operations stakeholders. During this review time the stakeholders would decide the appropriate actions (i.e., continue to monitor or identify new hazards or ineffective risk control) best suited for the operation. Once the meeting is concluded and the action items are addressed. The management level review meeting would occur during the second or third week of the month. At this review session, the Safety Manager would present the outcomes from the monthly analysis and assessment meeting to receive final approval or edits prior to the new or edited policy and procedures when living into the operation. The overall review process would start again. This ensures the operation is meeting the continuous improvement and monitoring requirements of the SMS program. While verifying organizational change management dimensions are being met to meet the most effective and sustainable amount of change within the operation.

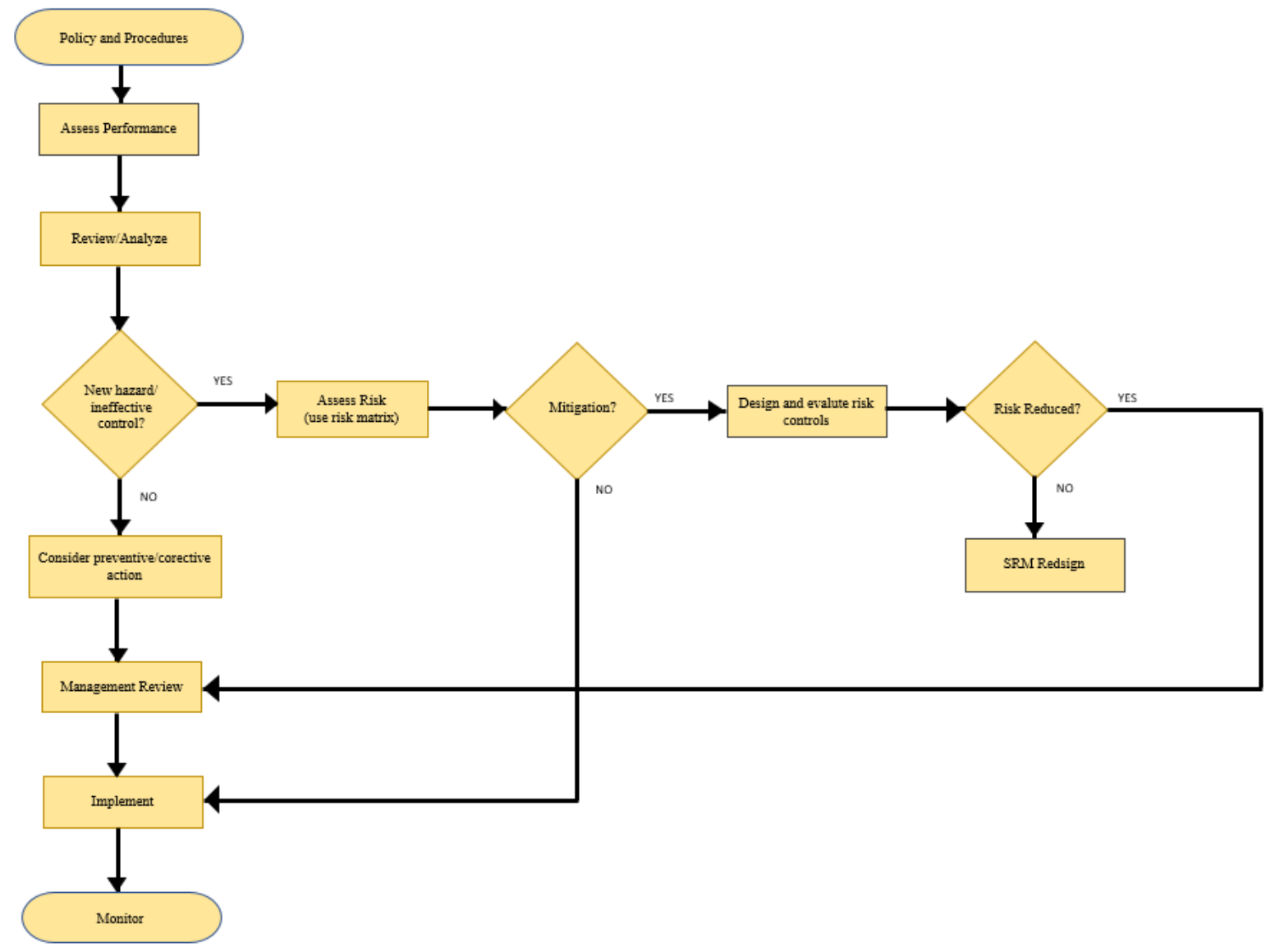

Figure 1. Safety Assurance Process Map 


\section{Safety Promotion}

The Safety Communication Checklist is designed to facilitate the needs of the Safety Promotion component of SMS while utilizing the Emotions dimension of organizational change management. The safety promotion component is the most vital when it comes to safety culture and receiving the "buy-in" from individuals within an organization and speaks how it is linked to the Emotions of organizational change management dimension.

Ad hoc communications are needed when various risk levels of events occur within the operation. This is more reactive communication needs within an operation. The weekly summarizing of operational safety concerns helps keep safety a focus and embeds concern within the operation itself. The monthly efforts are a part of the proactive measures of the SMS. Continuous improvement efforts build towards maintaining a positive safety culture. The yearly recommendations are embedded as part of the SMS requirements of the regulations. This tool was built in mind to provide communication direction for the Safety Manager of the operation.

The three tools (SRM Documentation Tool, SA Process Map, and the Safety Communication Checklist) are created to embrace the three dimensions of organizational change management, aiding the leaders to adopt to a magnitude of different changes or initiatives while following the same blueprint consistently that promotes a positive safety culture and identifies new hazards or ineffective risk controls within that operation. The SRM Documentation Tool is a step-by-step project plan that identifies milestones to achieve a consistent risk assessment throughout an operation. The document facilitates achieving the outcomes to make business decisions and involves stakeholders who have strong influence and power within an organizational structure. The documents also could be used as a promotion of how a decision was made, enabling the people and culture to adapt to the changes. SA Process Map is a blueprint in how to perform assessment processes and its life cycle within the Safety Assurance component. Lastly, the Safety Communication Checklist provides dynamic usability that addresses the third dimension of organizational change management directly by providing transparency and information updates to the employees that enable a positive safety culture. Developing these tools with Organizational Change Management principles to meet the SMS regulatory requirements provides certificate holders an execution plan but tools that will create sustainable and effective change in operation that will promote positive continuous improvement efforts that builds a robust safety culture.

\section{Application}

On April 12, 2019 the Purdue University Board of Trustees approved the purchase of thirteen single-engine Piper Archer aircraft. These aircraft were the replacement of the Cirrus trainer fleet which was designated as "aging and will require significant investment to maintain compliance with FAA regulations." (Purdue.edu). To manage the transition, the School of Aviation and Transportation Technology (SATT) complied with 14 CFR Part 5 Safety Management Systems (SMS) requirements on a voluntary basis. The integration of the SMS program was targeted to provide a comprehensive and preventive approach to manage safety hazards related to the transition of aircraft, Certified Flight Instructors (CFI) and students. During the aircraft transition, the three tools outlined in the report were used as primary tools to 
help ensure systematic, explicit, and comprehensive implementation that was applied consistently repeatedly when a new hazard or ineffective risk control is identified within the organization. These efforts were highlighted within safety communications, monthly review meetings and assessments on various operational levels, and implementation of new procedures.

\section{SRM Documentation Tool}

For the aircraft transition a Manual Fleet Approval Board and a subcommittee were created to complete the SRM requirements and work through the policy and procedure changes. The Manual Fleet Approval Board is made up of the Chief Flight Instructor (Flight Operations), Two Flight Faculty Members (Course Work), Director of Maintenance (Maintenance), and the Aviation Safety Manager. These members were the risk acceptors in their areas of responsibility related to hazards, mitigation plans, and overall functions of the Safety Risk Management (SRM) pillar. The SRM trigger for the fleet transition is listed as a primary example of the demonstration of section 5.51 in the 14 CFR Part 5 described in Advisory Circular 120 -92B. How the team complied with the five-part SRM requirement is by utilizing a subcommittee that identified and documented the;

- System descriptions

- Hazard identification

- Risk analysis

- Risk assessment

- Mitigation plans

○ Predicted residual risk

- Substitute risks

The subcommittee consisted of four full-time flight instructors (two Assistant Chiefs, Flight Instructors), seven students, which include traditional students and part-time flight instructors. The Board and the subcommittee met periodically to review the proposed changes, address edits and action items before the Board accepted the risk of each manual content change. Once the SRM process was completed and accepted by the risk acceptors the manual changes were completed and prepped for the "Ghost Manual" that was ultimately reviewed by the FAA and became an active manual revision in operation.

With any tool or program there are limitations to the usability of its design. The SRM Documentation Tool was designed in a way that was user-friendly for the stakeholders to share information. A pitfall of this mindset occurred when the facilitator was merging information with multiple hazards into one centralized document and it created an additional administrative workload for the facilitator. Since there were hundreds of hazards proactively identified, it became challenging to log them into the sample form. To mitigate that administrative pain point, the decision was made to keep the context of the form but migrate it into Excel to make it easier to manipulate the hazards and the related information.

\section{Safety Communication Checklist}

The Safety Communication Checklist was used primarily as a job-aid for the safety team to successfully meet the timeline requirements of each communication style established within 
the organization prior to the aircraft transition. A secondary use was utilizing the checklist as a training tool during the aircraft transition kickoff meeting that occurred during a safety meeting that included Instructors, Dispatchers, and Students. This assisted in providing the expectation of communication for each style of communication that would be sent out. This allowed the focus to be on the content and not be distracted in the format and actions required from each style of communication.

\section{Safety Assurance Process Map}

The primary functionality of the Safety Assurance Process Map was used for training purposes, and job-aid reference for understanding in what life cycle a hazard was currently in and assured the regulatory steps could be met efficiently since they were embedded in the process flow. This was helpful for the subcommittee because many people in the working group were not regularly attending management meetings and did not have the need to have a robust understanding of the administrative process flow. A limitation to this map was its non-interactive nature which left the current status interpretation to the stakeholder working the process. In order to overcome this limitation, the Aviation Safety Manager audited the process on a weekly basis.

\section{Conclusion}

Using these tools met compliance with the SMS regulatory requirements but also provided a pathway for Purdue University to implement safety practices with a solid framework to successfully comply and articulate the needs of the program to the active operation while verifying critical aspects were completed consistently that reduced confusion and missteps during this process of change management. The tools and processes utilized the three dimensions of organizational change management for effective and adopted changes within the operation. The results of this paper open new avenues for future research with applying and studying a certificate holder utilizing these tools in an active operation. Further quantitative research will provide gaps and possibly other tools needed within the safety policy component. 


\section{References}

Advisory Circular. (2015, January 01). Retrieved from:

https://www.faa.gov/documentLibrary/media/Advisory_Circular/AC_120-92B.pdf

Bareil, C. S. (2007). Patterns of discomfort with organizational change. Journal of Change Management, 1, 13-24.

Burnes, B. (2009). Managing Change. London: Prentice-Hall.

Duane A. Ludwig, C. R.-L. (2007). Safety Management Systems for Airports. Airport Cooperative Research Program (ACRP), $\mathrm{x}-1$. Retrieved from: https://www.google.com/books/edition/Safety_Management_Systems_for_Airports/5nqr $\mathrm{yVjratkC}$ ?hl=en\&gbpv=1\&dq=aviation+sms+safety+policy\&printsec=frontcover

GAM, (2015). Innovation-Based Change Management. Innovation, 7(32).

Green, E. C. (2020). Making Sense of Change Management (5th ed.). KoganPage.

Mark Hughes (2007) The Tools and Techniques of Change Management, Journal of Change Management, 7(1), 37-49, DOI: 10.1080/14697010701309435

Safety Management System. (2016, July 05). Retrieved from: https://www.faa.gov/about/initiatives/sms/explained/ 